\title{
Improvement of Bra Cleaning Technology Based on Mechanical Structure and Electronic Control Technology
}

\author{
Liangcheng Cheng, Yali Ruan, Zongye Chang \\ North China Electric Power University, Beinong Road, Huilongguan Town, \\ Changping District, Beijing, 102206, P.R. China
}

Keywords : Scanning cleaning, Anti deformation, Mechatronics.

\begin{abstract}
With the improvement of living standards, the requirements ofwomen's daily-wearing bra in the respect of comfort, appearance and cleanliness are also increasing. Traditional machine washing will destroy the bra shape, and cause the infection of mixed bacteria.Moreover, hand washing will occupy most of women's time. Therefore, a special bra cleaning machine can not onlyclean bra correctlybutalso save manpower.

This is a distinctive machine to washa piece of bra each time with both rinsing and dehydration function, which is combined with the 3D printer [1] scanning method to clean the bra. The unique design of supporting frame of bra can effectively prevent the deformation of the bra. Through the reasonable combination of mechanical structure and electronic control technology, the cleaning machine can realize the mechanical and electrical integration, and achieve effective cleaning.
\end{abstract}

\section{Project Background and Significance}

A bra is a common piece of underwear of women, mainly consisting of cups, straps, shoulder strap, buttons, with the function of supporting the bosom, increasing the comfort level and the motility when they participate in activities. It has gradually become the indispensable female clothing. As a piece of underwear to achieve beauty and comfort, a bra should be clean. The presence of dirt not only means impurities, bacteria, but also affects the permeability of the bra. At present, most of the cleaning methods include the hand washing andmachine washing. In the case of hand washing, bras can't be pushed too much for fear that the fabric be damaged. When it comes to bras with steel rings, they can't be twisted because it maycause the distortion of the bra structure.If the label is not marked that the clothing must be wash by hand, the vast majority of female consumers will choose to directly put the bra into the washing machine with other clothing, or use the laundry bag or cleaning ball.These cleaning methods can lead to a series of problems, like dyedbras,steel ring deformation, terrible cleaning effect and the damage of other clothing.

According to Guangzhou Carnation Breast Special Hospital's statistics, for women who regularly use washing machine to wash bra, the incidence of mastitis is higher than those who favor hand washingby $3 \sim 5$ times. The reason is that the clothes on the floss, thread and some very small fiber are easy to adhere to the brawhen the bra and other clothing are washed together. When women wear this kind of bra, those small fibers will stick to the nipple, and even block the tube infection, eventually causes mastitis.

In view of the importance of correctly cleaning bra, we designed a bra cleaning machine which is simple in operation, time-saving and labor-saving. 


\section{Product introduction}

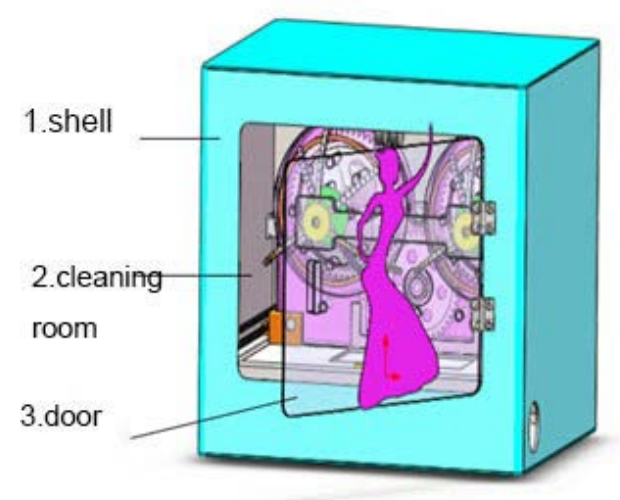

Fig. 1 shell

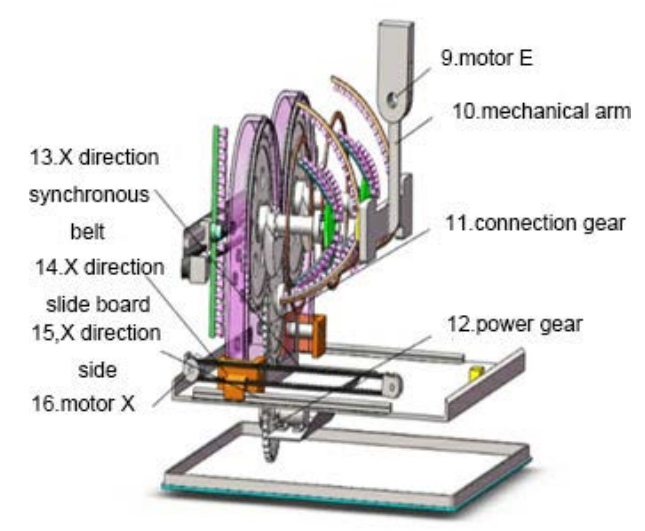

Fig.3 The internal structure $b$ of cleaning room $\mathrm{A}$

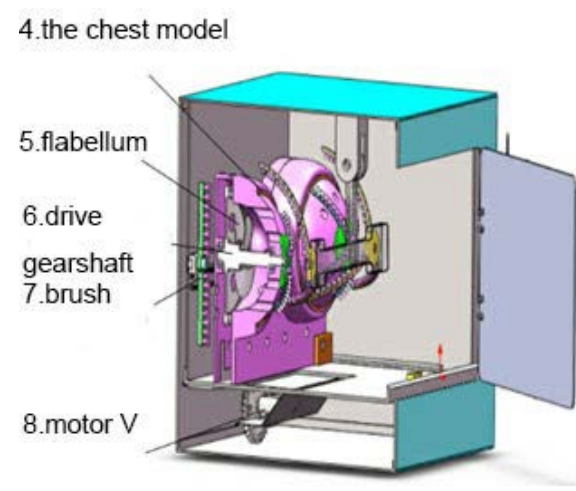

Fig.2 Cleaning room

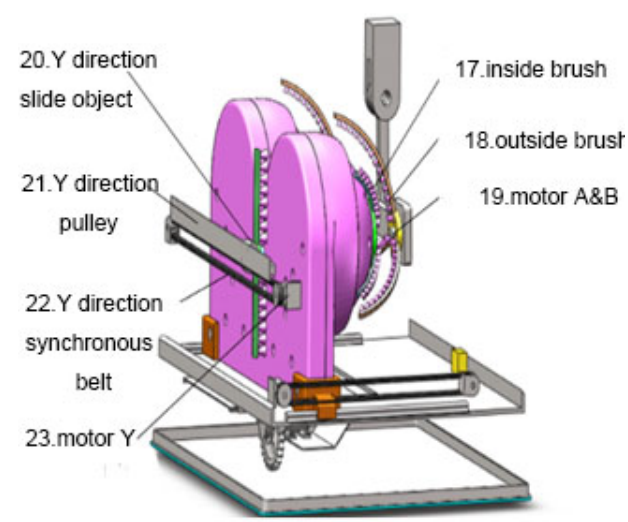

Fig.4 The internal structure $b$ of cleaning room $B$

\section{Washing Procedures (In Chronological Order)}

$\mathrm{X}$ motor's running, drives $\mathrm{X}$ direction of the synchronous belt running and makes bosom model and whole body bracket backward to the chest model back close to the Y direction back brush.

Y motor's running, scanning brush along the lateral reciprocating motion, cleansthe back.

V motor's running, transmits power to the gear shaft. At this point, the inside of the chest's brush begins rotating. And it is carried out to clean the inside of the bra cup. At the same time, blades begin to spin to make water flow in the box. Water penetrates the clothing, which enhanceswashing effects.

E motor's running, makes the mechanicalarm hem to the vertical position.

A \& B motors start work at the same time.The external brush starts rotating cleaning for bra's lateral cup.

E motor's running, makes the mechanical arm up to its original position.

\section{Instructions}

Open the front door, pull the body bracket, set bra above the chest model, and tie it up.

Pull the body bracket to the end, and shut the front door.

Plug in.

Press the open key to start automatic washing.[2] 

door.

End rings, open the front door, pull out the body bracket, remove the bra, and close the front

\section{The innovation points}

Just one washing each time. A cleaning machine, speciallydesigned for bra washing, avoids bacterial infection and hair bulb from other clothing in the process of cleaning. The lateral chest model can move to enclosure, facilitating the fix ofclothing. Scanning cleaning method combined with a pulsar washing way creates a new way for laundry. Overall structure of the product is small and light, which is suitable for home use.

\section{Part of program}

In order to achieve automatic control, we use the microcontroller STC12C5A60S2. Due to limited space, the following shows only part of the program:

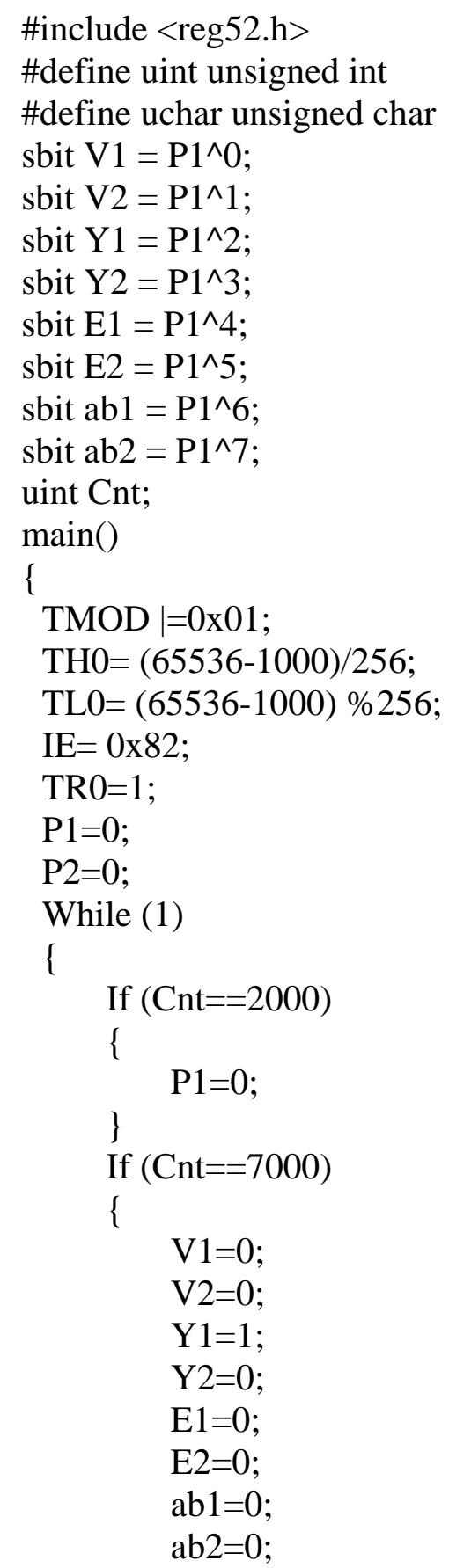




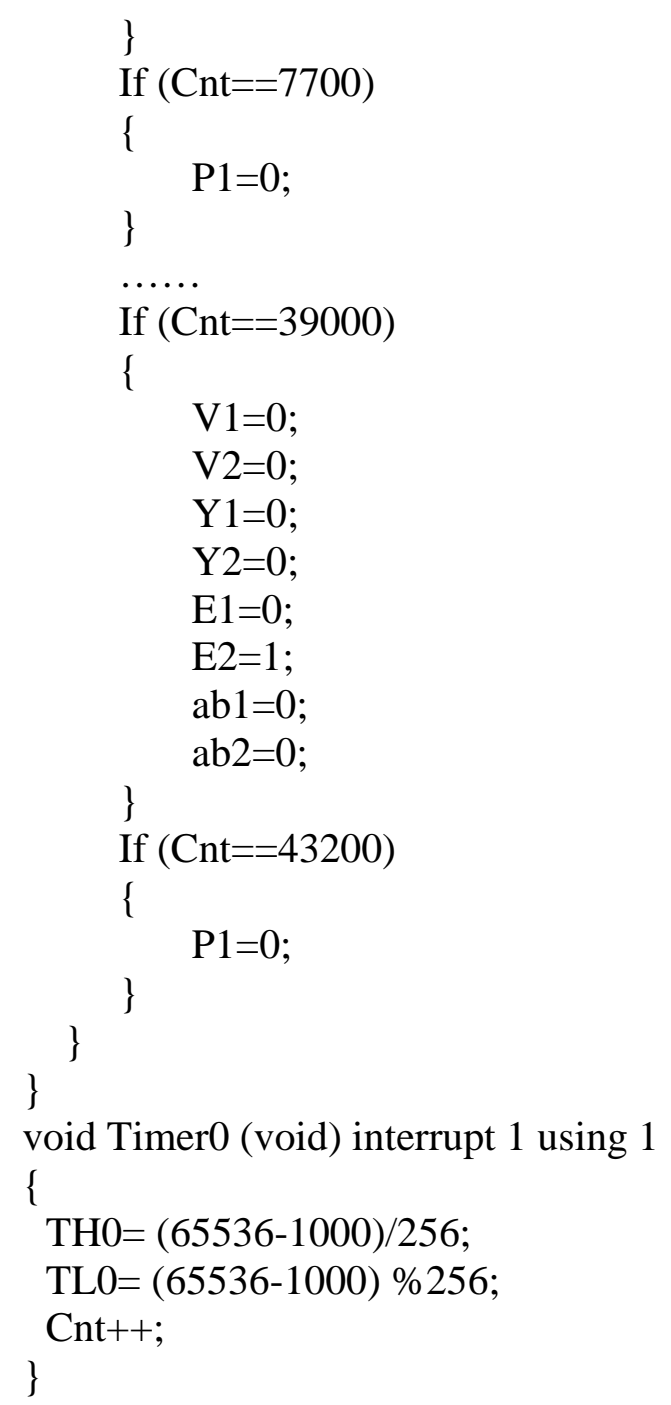

By downloading the program to the microcontroller, all the motors in the machine begin to run sequentially.

\section{Summary}

Based on 3D printing technology and concept, this bra cleaning machine achieves the effective integration of the mechanical design of cleaning brathrough the addition of internal and external front and rear side brush cleaning mode and the cooperation between the mechanical structure and electronic control devices. The bra supporting frame designed by the utility model can effectively prevent the distortion of the bra during the cleaning process. Step by stepcleaning can improve the cleaning efficiency, achieving all-round cleaning.

\section{References}

[1] Y.Y.Yang\& K.Zheng. Practical tutorial on mechanical 3D design (SolidWorks)[M / CD]. Beijing: Tsinghua University, 2013: 50-220.

[2] T.X.Guo. New concept note 51 monolithic machine C programming language-Improve, develop, and expand entry-level whole strategy. [M / CD]. Beijing: publishing house of electronics industry, 2009: 120-160. 